\title{
Review
}

\section{Future directions of resveratrol research}

\author{
Devin Wahl ${ }^{\mathrm{a}, \mathrm{b}}$, Michel Bernier ${ }^{\mathrm{c}}$, Stephen J. Simpson ${ }^{\mathrm{a}, \mathrm{d}}$, Rafael de Cabo ${ }^{\mathrm{c}}$ \\ and David G. Le Couteur ${ }^{\mathrm{a}, \mathrm{b}, *}$ \\ ${ }^{a}$ Charles Perkins Centre, University of Sydney, Sydney, Australia \\ ${ }^{\mathrm{b}}$ Aging and Alzheimer's Institute, ANZAC Research Institute, Concord Clinical School/Sydney \\ Medical School, Concord, Australia \\ ${ }^{\mathrm{c}}$ Translational Gerontology Branch, National Institute on Aging, National Institutes of Health, \\ Baltimore, MD, USA \\ ${ }^{\mathrm{d}}$ School of Life and Environmental Sciences, University of Sydney, Sydney, Australia
}

\begin{abstract}
Resveratrol improves healthspan and lifespan in many organisms. Several different targets and mechanisms of action have been proposed to explain the beneficial effects of resveratrol on healthspan and longevity, including the activation of a family of proteins known as sirtuins and its action as a calorie restriction mimetic. In this mini-review, we discuss some of the most recent findings to date in the resveratrol field and suggest three areas of future research based on those results.
\end{abstract}

Keywords: Aging, polyphenol, lifespan, healthspan, clinical trials, metabolic health

For the past several decades a number of animal studies, meta-analyses, and recent human clinical trials have focused on the ability of resveratrol (RSV), a naturally occurring polyphenol compound, to combat a variety of diseases such as type-2 diabetes, cardiovascular disorders, neurodegeneration, and cancer (for a comprehensive summary, see [1]). The molecular and biological mechanisms by which RSV exerts positive effects on cardiometabolic health are still under investigation and have mainly been studied in vitro [2]. RSV has been suggested to exert positive benefits on healthspan and lifespan partly by acting as a calorie restriction (CR) mimetic $[3,4]$ and activator of a family of $\mathrm{NAD}^{+}$- dependent protein deacetylases known as sirtuins [5]. Although beyond the scope of this short review, there are several possible mechanisms of action and targets by which RSV confers cardiometabolic health benefits, which include reducing inflammation and autophagy, improving insulin sensitivity and glucose

\footnotetext{
${ }^{*}$ Corresponding author: Professor David G. Le Couteur, C22 Repatriation General, Concord, The University of Sydney, NSW 2006, Australia. E-mail: david.lecouteur@sydney.edu.au.
}

homeostasis, and upregulating mitochondrial biogenesis (for detailed reviews, see [6, 7]).

Interestingly, RSV appears to have better prolongevity effects in some organisms compared to others as evidenced by the fact that this polyphenol is most effective for extending longevity in yeast, worms, and killifish while mice and flies are not as responsive to treatment $[3,8]$. Although there is evidence to suggest that RSV supplementation is beneficial to improve markers of cardiometabolic health in rodents and non-human primates, the translational potential of RSV has been controversial. For example, it has been argued that dosing and effective concentrations of RSV in rodent studies are much higher than the ones used in clinical trials. Another key argument points to the poor bioavailability of RSV and stresses the fact that clinical evaluation of RSV effectiveness has been limited by the small number of participants enrolled in underpowered studies [9]. Given the conflicting nature of these results, the purpose of this small review is to outline several recent findings in the field of RSV research and suggest a targeted approach for future investigations by 
focusing on several key areas: (1) Establishing effective durations of RSV treatment; (2) investigating RSV's effects on vasculature; and (3) determining the common mechanisms between RSV supplementation and $\mathrm{CR}$.

One key area of study in the field of RSV research should be the elucidation of effective length(s) of RSV treatment. A critical appraisal of the literature reveals that the effective duration of RSV treatment, rather than the dosage per se, must be elucidated before further translation of animal studies into human trials. A recent meta-review highlighted the fact that small animals are commonly treated with RSV for longer periods than in humans (comparatively speaking), indicating the need for new investigations into the effects and tolerability of long-term RSV treatment in human volunteers [10]. Mice fed a high-fat diet and chronically exposed to dietary RSV supplementation — starting at middle age until their natural death - showed improvements in insulin sensitivity indices and health parameters similar to those observed in mice on standard diet, while simultaneously improving lifespan [11]. Whether long-term RSV supplementation will confer similar benefits to obese individuals remains to be determined.

There have been several investigations into the health outcomes of long-term RSV supplementation in Old World nonhuman primates (Macaca mulatta) consuming a high-fat, high-sugar diet. Beneficial changes in several biochemical, molecular, and histological markers were observed following a 2-year RSV treatment in middle-aged primates fed an obesogenic diet. Specifically, there was a reduction in brain inflammation, improvements in microvasculature integrity, and refined neurotransmission [12, 13]. Improvements of several metabolic outcomes were also found, such as restored islet cell physiology [14], insulin signaling in adipose tissues [15], and a significant prevention of aortic arterial stiffness caused by consumption of the high-fat, high-sugar diet [16]. These results suggest that long-term RSV supplementation is an effective and well-tolerated intervention to improve markers of cardiac and metabolic health in a close human relative.

There is limited information on the effects of long-term RSV supplementation is humans because most clinical trials to date have lasted less than 6 months in duration. For example, Berman et al. [17] recently detailed clinical trials assessing the therapeutic potential of RSV in cancers, cardiovascular disease, diabetes, and non-alcoholic fatty liver disease (NAFLD). Out of the 23 clinical trials investigated, only 3 had protocols that lasted longer than 6 months, while most were 1 to 3 months in duration. One recent meta-analysis focused on the aspect of length of treatment in humans by reporting clinical trials that lasted for more than 6 months. Male and female patients with metabolic syndrome saw significant improvement in plasma adiponectin, but not leptin, levels after RSV treatment for a period $\geq 6$ months [18]. Long-term (12-months) RSV supplementation has been found to boost brain health in humans by decreasing $A \beta 40$ and $A \beta 42$ levels in cerebrospinal fluid while simultaneously improving memory as measured by Mini-Mental State Examination (MMSE) scores [19].

There have been many clinical studies examining the role of RSV in diabetes, particularly by looking at markers of insulin sensitivity and glucose tolerance. As indicated by Szkudelski and Szkudelski, most of the trials showed no effect and lasted less than 6 months [20]. Nevertheless, non-human primates on an obesogenic diet showed significant reduction in central arterial wall stiffness in response to long-term RSV supplementation [16]. There is now emerging evidence that RSV may partly contribute to improvements in several health measures in humans by improving vasculature integrity [21]. For example, RSV treatment led to an increase in neurovascular coupling capacity and improved memory in older adults [22]. Furthermore, ingestion of $125 \mathrm{mg} \mathrm{RSV} /$ day for 6 months improved 6-minute walking performance in older adults with peripheral artery disease (PAD) [23], although the authors concluded that the results did not quite reach clinical significance. Strikingly, short-term RSV supplementation for 6 weeks increased mitochondrial number and genetic markers of improved muscle vasculature function in older adults with impaired glucose tolerance, without alterations in insulin sensitivity and glucose metabolism [24]. Given those positive results, we suggest that the mechanisms by which RSV improves vascular health might be a more focused direction of study until additional and more robust health benefits are confirmed in humans (i.e., improving the diabetic phenotype).

Earlier in-vitro data suggested that RSV is a potent activator of the enzyme sirtuin 1 (SIRT1). CR also activates SIRT1 and is considered one of the most robust, non-genetic nutritional interventions to improve healthspan and lifespan from yeast to 
non-human primates [25]. Although impractical, there is emerging evidence to suggest that CR might be effective in humans [26]; however, a severe CR diet is difficult to maintain, especially in western societies, where rates of obesity are constantly rising due to high consumption of calorie-dense foods [27]. Despite the development of other sirtuin activators with overlapping CR-like properties (i.e., SRT2104, SRT1720) [28, 29], the fact remains that RSV is one of the most potent and well-studied CR mimetic compounds [7, 30]. For example, obese humans on 30-day RSV supplementation showed a significant increase in muscle SIRT1 levels, improved mitochondrial respiration, and increased insulin sensitivity, consistent with short-term CR-mimetic effects [31]. CR [32] and prolonged RSV supplementation are two effective interventions that promote longevity and cardiometabolic health in non-human primates, but it is unclear whether similar results will be achieved in humans. Therefore, in order to provide new insights into the impact of nutritional interventions on human health, one needs to further elucidate the common mechanisms of action between CR and long-term RSV supplementation.

To conclude, the positive effects of RSV on metabolic health highlights the need for continued clinical research, even though adverse responses of RSV supplementation in humans have been reported. This underscores the challenges of translating results from animal studies and preliminary findings to more stringent clinical trials $[1,33]$. The results from rodent and non-human primate studies suggest that longer durations were the most effective with regard to lifespan extension and comprehensive measures of health in response to RSV. Furthermore, there is a need for improved understanding of the effects of RSV on vascular health given the positive results seen in mice and non-human primates. Finally, it is imperative to continue to study the similarities between long-term RSV supplementation and CR in humans.

\section{Funding}

This work was supported by the Aging and Alzheimer's Research Institute, National Health and Research Council of Australia (NHMRC) grant numbers \#571328, \#1084267. MB and RdC were supported by the Intramural Program of the National Institute on Aging, NIH. DW was partly supported by the American Australian Association (AAA) Education Fund.

\section{References}

[1] Novelle MG, Wahl D, Dieguez C, Bernier M, de Cabo R. Resveratrol supplementation: Where are we now and where should we go? Ageing Research Reviews. 2015;21:1-15.

[2] Baur JA, Sinclair DA. Therapeutic potential of resveratrol: The in vivo evidence. Nature Reviews Drug Discovery. 2006;5(6):493-506.

[3] Pallauf K, Rimbach G, Rupp PM, Chin D, Wolf IM. Resveratrol and lifespan in model organisms. Current Medicinal Chemistry. 2016;23(41):4639-80.

[4] Martin B, Mattson MP, Maudsley S. Caloric restriction and intermittent fasting: Two potential diets for successful brain aging. Ageing Research Reviews. 2006;5(3):332-53.

[5] Bonkowski MS, Sinclair DA. Slowing ageing by design: The rise of $\mathrm{NAD}(+)$ and sirtuin-activating compounds. Nature Reviews Molecular Cell Biology. 2016;17(11):679-90.

[6] Britton RG, Kovoor C, Brown K. Direct molecular targets of resveratrol: Identifying key interactions to unlock complex mechanisms. Annals of the New York Academy of Sciences. 2015;1348(1):124-33.

[7] Baur JA. Resveratrol, sirtuins, and the promise of a DR mimetic. Mechanisms of Ageing and Development. 2010; 131(4):261-9.

[8] Hector KL, Lagisz M, Nakagawa S. The effect of resveratrol on longevity across species: A meta-analysis. Biology Letters. 2012;8(5):790-3.

[9] Visioli F. The resveratrol fiasco: Pharmacological Research. 2014;90:87.

[10] Fernandez-Quintela A, Carpene C, Fernandez M, Aguirre L, Milton-Laskibar I, Contreras J, Portillo MP. Anti-obesity effects of resveratrol: Comparison between animal models and humans. Journal of Physiology and Biochemistry. 2016;15:15.

[11] Baur JA, Pearson KJ, Price NL, Jamieson HA, Lerin C, Kalra A, Prabhu VV, Allard JS, Lopez-Lluch G, Lewis K, Pistell PJ, Poosala S, Becker KG, Boss O, Gwinn D, Wang M, Ramaswamy S, Fishbein KW, Spencer RG, Lakatta EG, Le Couteur D, Shaw RJ, Navas P, Puigserver P, Ingram DK, de Cabo R, Sinclair DA. Resveratrol improves health and survival of mice on a high-calorie diet. Nature. 2006;444(7117): 337-42.

[12] Bernier M, Wahl D, Ali A, Allard J, Faulkner S, Wnorowski A, Sanghvi M, Moaddel R, Alfaras I, Mattison JA, Tarantini S, Tucsek Z, Ungvari Z, Csiszar A, Pearson KJ, de Cabo R. Resveratrol supplementation confers neuroprotection in cortical brain tissue of nonhuman primates fed a high-fat/sucrose diet. Aging (Albany, NY). 2016;8(5):899-916.

[13] Swomley AM, Triplett JC, Keeney JT, Warrier G, Pearson KJ, Mattison JA, de Cabo R, Cai J, Klein JB, Butterfield DA. Comparative proteomic analyses of the parietal lobe from rhesus monkeys fed a high-fat/sugar diet with and without resveratrol supplementation, relative to a healthy diet: Insights into the roles of unhealthy diets and resveratrol on function. Journal of Nutritional Biochemistry. 2017;39: 169-79. 
[14] Fiori JL, Shin YK, Kim W, Krzysik-Walker SM, GonzalezMariscal I, Carlson OD, Sanghvi M, Moaddel R, Farhang K, Gadkaree SK, Doyle ME, Pearson KJ, Mattison JA, de Cabo R, Egan JM. Resveratrol prevents beta-cell dedifferentiation in nonhuman primates given a high-fat/high-sugar diet. Diabetes. 2013;62(10):3500-13.

[15] Jimenez-Gomez Y, Mattison JA, Pearson KJ, MartinMontalvo A, Palacios HH, Sossong AM, Ward TM, Younts CM, Lewis K, Allard JS, Longo DL, Belman JP, Malagon MM, Navas P, Sanghvi M, Moaddel R, Tilmont EM, Herbert RL, Morrell CH, Egan JM, Baur JA, Ferrucci L, Bogan JS, Bernier M, de Cabo R. Resveratrol improves adipose insulin signaling and reduces the inflammatory response in adipose tissue of rhesus monkeys on high-fat, high-sugar diet. Cell Metabolism. 2013;18(4):533-45.

[16] Mattison JA, Wang M, Bernier M, Zhang J, Park SS, Maudsley S, An SS, Santhanam L, Martin B, Faulkner S, Morrell C, Baur JA, Peshkin L, Sosnowska D, Csiszar A, Herbert RL, Tilmont EM, Ungvari Z, Pearson KJ, Lakatta EG, de Cabo R. Resveratrol prevents high fat/sucrose diet-induced central arterial wall inflammation and stiffening in nonhuman primates. Cell Metabolism. 2014;20(1):183-90.

[17] Berman AY, Motechin RA, Wiesenfeld MY, Holz MK. The therapeutic potential of resveratrol: A review of clinical trials. NPJ Precision Oncology. 2017;1:35.

[18] Mohammadi-Sartang M, Mazloom Z, Sohrabi Z, Sherafatmanesh S, Barati-Boldaji R. Resveratrol supplementation and plasma adipokines concentrations? A systematic review and meta-analysis of randomized controlled trials. Pharmacological Research. 2017;117:394-405.

[19] Moussa C, Hebron M, Huang X, Ahn J, Rissman RA, Aisen PS, Turner RS. Resveratrol regulates neuro-inflammation and induces adaptive immunity in Alzheimer's disease. Journal of Neuroinflammation. 2017;14(1):1.

[20] Szkudelski T, Szkudelska K. Resveratrol and diabetes: From animal to human studies. Biochimica et Biophysica Acta. 2015;1852(6):1145-54.

[21] Csiszar A. Anti-inflammatory effects of resveratrol: Possible role in prevention of age-related cardiovascular disease. Annals of the New York Academy of Sciences. 2011;1215: $117-22$.

[22] Wong RH, Raederstorff D, Howe PR. Acute resveratrol consumption improves neurovascular coupling capacity in adults with type 2 diabetes mellitus. Nutrients. 2016;8(7).

[23] McDermott MM, Leeuwenburgh C, Guralnik JM, Tian L, Sufit R, Zhao L, Criqui MH, Kibbe MR, Stein JH, LloydJones D, Anton SD, Polonsky TS, Gao Y, de Cabo R, Ferrucci L. Effect of resveratrol on walking performance in older people with peripheral artery disease: The RESTORE randomized clinical trial. JAMA cardiology. 2017;2(8):902-7.
[24] Pollack RM, Barzilai N, Anghel V, Kulkarni AS, Golden A, O’Broin P, Sinclair DA, Bonkowski MS, Coleville AJ, Powell D, Kim S, Moaddel R, Stein D, Zhang K, Hawkins $\mathrm{M}$, Crandall JP. Resveratrol improves vascular function and mitochondrial number but not glucose metabolism in older adults. The Journals of Gerontology Series A, Biological Sciences and Medical Sciences. ;72(12):1703-9.

[25] Picca A, Pesce V, Lezza AMS. Does eating less make you live longer and better? An update on calorie restriction. Clinical Interventions in Aging. 2017;12:1887-902.

[26] Most J, Tosti V, Redman LM, Fontana L. Calorie restriction in humans: An update. Ageing Research Reviews. 2017;39: 36-45.

[27] Drewnowski A. Nutrient density: Addressing the challenge of obesity. The British Journal of Nutrition. 2017:1-7.

[28] Mercken EM, Mitchell SJ, Martin-Montalvo A, Minor RK Almeida M, Gomes AP, Scheibye-Knudsen M, Palacios HH, Licata JJ, Zhang Y, Becker KG, Khraiwesh H, GonzálezReyes JA, Villalba JM, Baur JA, Elliott P, Westphal C, Vlasuk GP, Ellis JL, Sinclair DA, Bernier M, de Cabo R. SRT2104 extends survival of male mice on a standard diet and preserves bone and muscle mass. Aging Cell. 2014;13(5):787-96.

[29] Mitchell SJ, Martin-Montalvo A, Mercken EM, Palacios HH, Ward TM, Abulwerdi G, Minor RK, Vlasuk GP, Ellis JL, Sinclair DA, Dawson J, Allison DB, Zhang Y, Becker KG, Bernier M, de Cabo R. The SIRT1 activator SRT1720 extends lifespan and improves health of mice fed a standard diet. Cell Reports. 2014;6(5):836-43.

[30] Allard JS, Perez E, Zou S, de Cabo R. Dietary activators of Sirt1. Molecular and Cellular Endocrinology. 2009;299(1): 58-63.

[31] Timmers S, Konings E, Bilet L, Houtkooper RH, van de Weijer T, Goossens GH, Hoeks J, van der Krieken S, Ryu D, Kersten S, Moonen-Kornips E, Hesselink MKC, Kunz I, Schrauwen-Hinderling VB, Blaak E, Auwerx J, Schrauwen P. Calorie restriction-like effects of 30 days of resveratrol supplementation on energy metabolism and metabolic profile in obese humans. Cell Metabolism. 2011;14(5):612-22.

[32] Mattison JA, Colman RJ, Beasley TM, Allison DB, Kemnitz JW, Roth GS, Ingram DK, Weindruch R, de Cabo R, Anderson RM. Caloric restriction improves health and survival of rhesus monkeys. Nature Communications. 2017;8:14063.

[33] Smoliga JM, Colombo ES, Campen MJ. A healthier approach to clinical trials evaluating resveratrol for primary prevention of age-related diseases in healthy populations. Aging (Albany NY). 2013;5(7):495-506. 